\title{
Alternate Solutions Analysis For Transportation Problems
}

Veena Adlakha, University of Baltimore, USA

Krzysztof Kowalski, Connecticut Department of Transportation, USA

\begin{abstract}
The constraint structure of the transportation problem is so important that the literature is filled with efforts to provide efficient algorithms for solving it. The intent of this work is to present various rules governing load distribution for alternate optimal solutions in transportation problems, a subject that has not attracted much attention in the current literature, with the result that the load assignment for an alternate optimal solution is left mostly at the discretion of the practitioner. Using the Shadow Price theory we illustrate the structure of alternate solutions in a transportation problem and provide a systematic analysis for allocating loads to obtain an alternate optimal solution. Numerical examples are presented to explain the proposed process.
\end{abstract}

Keywords: transportation problem, shadow prices, linear programming

\section{INTRODUCTION}

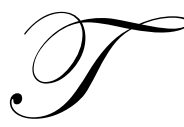

he transportation problem (TP) deals with the distribution of goods from several supply points (sources) to a number of demand points (destinations). When addressing a TP, the practitioner usually has a given capacity at each supply point and a given requirement at each demand point. Many decision problems, such as production inventory, job scheduling, production distribution, and investment analysis, can be formulated as TPs. Good financial decisions concerning facility location also attempt to minimize total transportation and production costs for the entire system.

It is well known that a TP can be formulated as a linear program and solved by the regular simplex (big-M), the dual simplex method, or even an interior approach method. The literature is filled with efforts to provide effective techniques for solving a TP. The stepping-stone algorithm (SS) has proven very successful and has become the standard technique for several decades. Many researchers have provided techniques for overcoming major obstacles of the SS algorithm, such as difficulties in identifying an initial basic feasible solution, resolving SS degeneracy, and enumerating SS paths [3, 4, 6-8]. Other researchers have provided a new outlook for the solution with other algorithms [5, 9]. Adlakha and Kowalski [1] provided a novel algorithm for solving the transportation problem based on the theory of absolute points. The algorithm has a limited application to only those problems where absolute points can be identified.

While the literature on the TP is abundant, discussion of alternate optimal solutions (AOSs) is very limited. Frequently in applications, there are more than two alternate optimal solutions. Standard TP software packages deliver an optimal solution without any indication of the existence of alternate optimal solutions. Consequently, two different software packages will often return dissimilar (alternate) optimal solutions to the same TP, potentially causing confusion for students and practitioners alike. Even when there is an awareness of alternate optimal solutions, the determination of load assignment is left mostly at the discretion of the practitioner.

In this paper we discuss the development of AOSs based on the Shadow Price theory. We demonstrate that the distribution of AOSs is not at all random, but is governed by certain rules and easily can be determined through simple operations. We further provide an algorithm to develop alternate optimal solutions systematically for load distributions to include cells chosen from the specific list. In practice a decision maker wants to know if it is possible to use his/her "favorite" route. The proposed algorithm identifies the inherent structure of the AOSs to 
determine commonality in these solutions by identifying the fixed cells, thereby empowering a practitioner with flexibility to allocate loads strategically on the basis of an analysis of alternate assignments.

\section{THE TRANSPORTATION PROBLEM}

The transportation problem can be stated as a distribution problem in which there are $m$ suppliers (sources) and $n$ customers (destinations). Each of the $m$ suppliers can ship to any of the $n$ customers at a shipping cost per unit $c_{i j}$ (unit cost for shipping from supplier $i$ to customer $j$ ). Each supplier has $a_{i}$ units of supply, $1 \leq i \leq m$, and each customer has a demand of $b_{j}$ units, $1 \leq j \leq \mathrm{n}$. The objective is to determine which routes are to be opened and the size of the loads/shipment on those routes, so that the total cost of meeting demand, given the supply constraints, is minimized.

\section{TP formulation:}

$\begin{array}{ll}\operatorname{minimize} & Z=\sum_{i=1}^{m} \sum_{j=1}^{n} c_{i j} x_{i j} \\ \text { subject to } & \sum_{j=1}^{n} x_{i j}=a_{i} \text { for } i=1,2, \ldots, m\end{array}$

$\sum_{i=1}^{m} x_{i j}=b_{j}$ for $j=1,2, \ldots, n$

$x_{i j} \geq 0$ for all $(i, j)$

Without loss of generality, we assume that

$$
\begin{aligned}
& \sum_{i=1}^{m} a_{i}=\sum_{j=1}^{n} b_{j} \\
& a_{i}, b_{j}, c_{i j} \geq 0
\end{aligned}
$$

From the Simplex theory approach to solving TPs, we know that there are $N=(m+n-1)$ basic variables in any given distribution, i.e., there are $(m+n-1)$ load assignments in the final optimal solution tableau. This rule, and a determination about a minimum possible number of loads (non-zero basic variables), can also be derived through another, much simpler analysis based on the widely known Northwest Corner Pattern (NWCP), shown in Figure 1 (assume $n>m$ ). The rule starts with a load at cell $(1,1)$ and then continues loading the TP matrix along corresponding rows or columns while meeting demand and supply constraints. Each load assignment results in introduction of either a new row or column with the total number of loads as $N=1+(m-1)+(n-1)=(m+n-1)$. Note that it is possible to rearrange the order of columns and rows in any loaded TP tableau to create a NWCP.

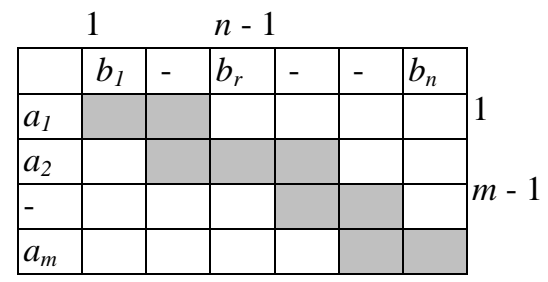

Figure 1. Typical Northwest Corner Pattern 
When $N$ is less than $(m+n-1)$, as shown in Figure 2, then the solution to TP is degenerate, which in turn means that some of the basic variables are equal to 0 .

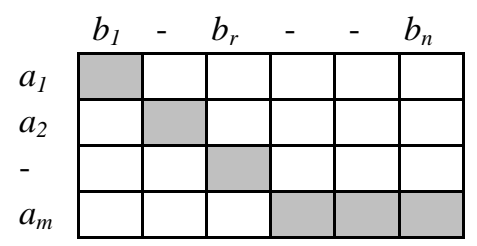

Figure 2. Degenerate Solution with NWCP

It might be the case in a TP that $N$ is greater than $(m+n-1)$. Such a situation reflects a presence of an AOS where some rows/columns can be simultaneously loaded. This phenomenon has two cases: simple SS chains as shown in Figure 3 or complex SS chains as shown in Figure 4. An explanation of this phenomenon based on the theory of absolute points was provided by Adlakha and Kowalski [1]. In the Simplex analysis, such a situation corresponds with the presence of non-basic variables with cost coefficients equal to zero. If more than one optimal solution exists, then many AOSs exist. Any feasible positive-weighted average of the two basic optimal solutions also yields an AOS. The resultant set of variables does not constitute a basis and contains more variables than the number of constraining relations, $(m+n-1)$.

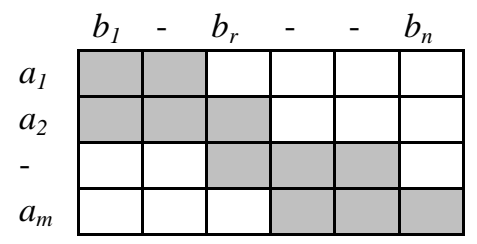

Figure 3. NWCP with AOS involving simple SS chains.

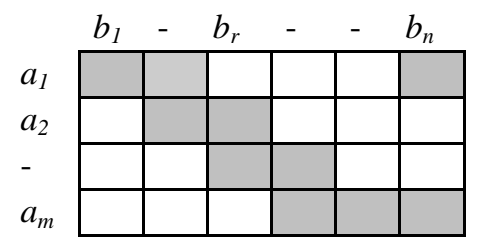

Figure 4. NWCP with AOS with complex SS chains.

It must be noted that there can also be a distribution comprising a combination of all three situations depicted in Figures 1, 2, 3 and 4.

\subsection{Shadow prices}

The process of calculating shadow prices requires that we define a dual price $\mathrm{u}_{\mathrm{i}}$ for each supply constraint and a dual price $v_{j}$ for each demand constraint. Computing these $u_{i}$ and $v_{j}$ requires that for each basic cell the cost coefficient $c_{i j}$ be equal to $\left(u_{i}+v_{j}\right)$. By setting any one $u_{i}=0$ or $v_{j}=0$, one can solve the system of equations for all remaining $u_{i}$ and $v_{j}$ values. For each nonbasic cell, the net evaluation index, $c_{i j}-\left(u_{i}+v_{j}\right)$, provides the incremental change in the total cost that will be obtained by allocating one unit of flow to the corresponding cell. For an optimal solution, we use the term shadow price $(\mathrm{SP})$ for $\left(\mathrm{u}_{\mathrm{i}}+\mathrm{v}_{\mathrm{j}}\right)$ values and present these as a shadow price matrix $(\mathrm{SP}$ 
matrix). It is obvious that all shadow prices for all nonbasic cells are less than the corresponding cost coefficients $c_{\mathrm{ij}}$.

Definition 1: A shadow price in the SP matrix is called an optimal shadow price (optimal SP) if it equals the corresponding unit cost coefficient $\mathrm{c}_{\mathrm{ij}}$.

By construction, the shadow prices for all basic cells are optimal SPs. We analyze the SP matrix to identify the existence of AOSs by locating nonbasic cells with optimal SPs. These cells represent the locations where loads may be moved to obtain an AOS.

\section{ALTERNATE SOLUTION ANALYSIS}

A shadow price analysis of the optimal solution leads us to a situation from either Figure 1, 3, or 4. Note that the situation in Figure 2 prohibits the creation of an SP matrix. In such a case, dummy variables need to be introduced to bring the number of variables to $(m+n-1)$. A systematic method for determining those dummy locations is presented by Adlakha et al. [2].

Definition 2: A basic cell is referred to as fixed due to the absolute structure of the TP if it is loaded with the exact same amount in any optimal solution.

Note that a cell $(i, j)$ will be considered fixed due to the absolute structure of the TP only if there is only one optimal SP present in a given row/column of the SP matrix. In this situation the cell must always be loaded with $\min \left(\mathrm{a}_{\mathrm{i}}, \mathrm{b}_{\mathrm{j}}\right)$ in any optimal solution. At the start of AOS analysis, such cell $(i, j)$ is identified and loaded with $\mathrm{a}_{\mathrm{i}}$ or $\mathrm{b}_{\mathrm{j}}$. The corresponding satisfied row (or column) is eliminated from further consideration and the corresponding demand (or supply) is adjusted. This operation can (but need not) create a new 'one optimal SP' row or column in the revised SP matrix. After depleting all 'one optimal SP' rows and columns, all identified values are recorded as fixed due to the absolute structure of the TP. The reduced SP matrix is carried on for further analysis.

Definition 3: A cell is fixed due to the constraint structure of the TP if it is always to be loaded with at least some minimum specified amount in any optimal solution.

Note that a cell $(i, j)$ will be considered to be fixed due to the constraint structure of the TP when multiple optimal SPs are present in row $i$ or column $j$ of the SP matrix.

\subsection{A pre-screening algorithm}

We introduce a pre-screening algorithm to identify cells which are fixed due to the constraint structure of the TP. Consider the reduced SP matrix after deleting the rows/columns related to the cells fixed due to the absolute structure of the TP. We want to determine the minimum amount of load, $\mathrm{X}_{i j}$, which must appear in every possible AOS. To determine this amount, analyze every row and column of the reduced optimal SP matrix to determine a loaded cell, $(s, t)$, where $\sum_{i \neq s} a_{i}<b_{t}$ or $\sum_{j \neq t} b_{j}<a_{s}$. Note that only loaded cells are considered in verifying this relationship. After identifying cell $(s, t)$, the value of $\mathrm{X}_{\mathrm{st}}$ is set as follows:

$\mathrm{X}_{s t}=b_{t}-\sum_{i \neq s} a_{i} \quad$ or $\quad X_{s t}=a_{s}-\sum_{j \neq t} \mathrm{~b}_{\mathrm{j}}$

All loads $\mathrm{X}_{s t}$ identified by the equalities (4) are recorded as fixed due to the constraint structure of the TP, and are subtracted from the corresponding supply and demand values. The above operations lead to the following algorithm. 


\subsection{An alternate optimal solutions algorithm}

To identify and develop alternate optimal solutions in a TP, the following algorithm is proposed:

Step 1. Solve the TP using any method to get an optimal solution.

Step 2. Develop the SP matrix. If all SP values for the nonbasic (not currently loaded) cells are less than the corresponding cost coefficients, no AOS exist. Otherwise, continue.

Step 3. Search for a fixed due to the absolute structure of the TP cell. If none, go to Step 5.

Step 4. Assign maximum possible load at the selected cell and delete the corresponding row/column while adjusting the demand/supply for the associated column/ row. Go to Step 3 with the reduced load matrix.

Step 5. Consider the current load matrix and set the loads for all cells corresponding to remaining optimal SPs as 0. We refer to this matrix as the pre-screening load matrix.

Step 6. Perform pre-screening analysis on this load matrix to determine cells that are fixed due to the constraint structure of the TP. If none, go to Step 8.

Step 7. Load all locations identified in Step 6 as determined by Equation (4) and adjust the corresponding demands and supplies. Go to Step 6.

Step 8. Assign the remaining loads in any manner within the constraints of supply and demand to obtain an alternate optimal solution.

As a result of this algorithm we obtain the fixed part of the solution identified in Steps 4 and 7. The loads remaining to be assigned after Step 7 constitute a floating part of the solution.

\section{NUMERICAL EXAMPLE}

In this section we present a detailed example to illustrate the steps of the proposed alternate optimal solutions algorithm.

Table 1. Cost Matrix for the Numerical Example

$a_{1}$
$a_{2}$
$a_{3}$
$a_{4}$

Demand

\begin{tabular}{|c|c|}
\hline $\mathrm{b}_{1}$ & \\
\hline 2 & \\
\hline 3 & \\
\hline 5 & \\
\hline 7 & \\
\hline 50 & \\
\hline
\end{tabular}

\begin{tabular}{|c|c|}
\hline $\mathrm{b}_{2}$ & \\
\hline 1 & \\
\hline 2 & \\
\hline 4 & \\
\hline 5 & 5 \\
\hline 30 & 30 \\
\hline
\end{tabular}

\begin{tabular}{|c|c}
\hline $\mathrm{b}_{3}$ & $\mathrm{~b}$ \\
\hline 3 & 2 \\
\hline 1 & \\
\hline 2 & 3 \\
\hline 5 & 50 \\
\hline 30 & \\
\hline
\end{tabular}

Step 1: An optimal non-degenerate solution of this TP using the Management Scientist software with a total cost of $\$ 390$ is obtained as follows:

Table 2. Optimal Solution for the Numerical Example

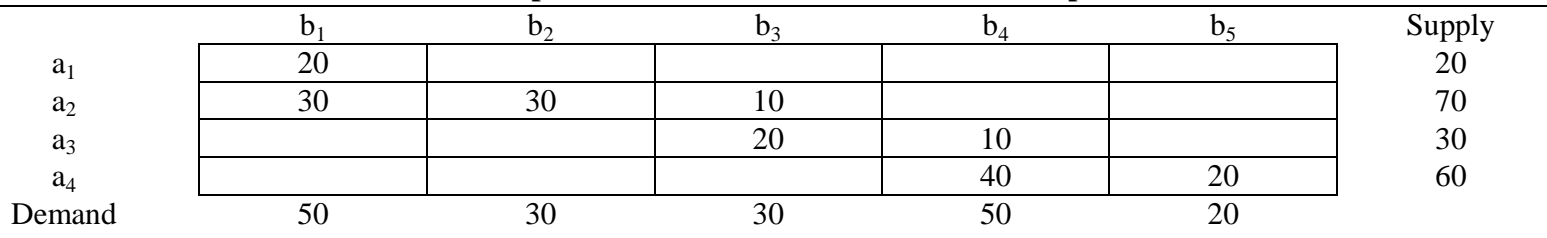

Step 2: The SP matrix is presented in Table 3. The shadow prices corresponding to the optimal solution are marked by *. Two additional optimal SPs are marked by superscript ${ }^{\text {a }}$. 
Table 3. The SP Matrix for the Numerical Example

\begin{tabular}{|c|c|c|c|c|c|}
\hline & $\mathrm{v}_{1}$ & $\mathrm{v}_{2}$ & $\mathrm{v}_{3}$ & $\mathrm{v}_{4}$ & $\mathrm{v}_{5}$ \\
\hline $\mathrm{u}_{1}$ & $2 *$ & $\mathbf{1}^{\mathrm{a}}$ & 0 & -1 & -3 \\
\hline $\mathrm{u}_{2}$ & $3 *$ & $2 *$ & $1 *$ & 0 & -2 \\
\hline $\mathrm{u}_{3}$ & 4 & 3 & $2 *$ & $1 *$ & -1 \\
\hline $\mathrm{u}_{4}$ & 6 & $5^{a}$ & 4 & $3 *$ & $1 *$ \\
\hline $\mathrm{v}_{\mathrm{j}}$ & 6 & 5 & 4 & 3 & 1 \\
\hline
\end{tabular}

There are two non-basic cells, $(1,2)$ and $(4,2)$ with optimal SPs (the SP values equal to the corresponding costs of Table 1), thus indicating the presence of an AOS. An alternate solution can be obtained by using a simple SS chain starting at cell $(1,2)$ and/or a complex SS chain starting at cell $(4,2)$.

Step 3: Column 5 has only one SP. Therefore, basic cell $(4,5)$ is fixed due to the absolute structure of the TP. Step 4: Assign $\mathrm{X}_{45}=\min \left(\mathrm{a}_{4}, \mathrm{~b}_{5}\right)=20$. Delete Column 5 and adjust $\mathrm{a}_{4} \rightarrow 40$. There is no other cell which is fixed due to the absolute structure of the TP.

Step 5: The reduced load matrix with loads of 0 assigned to cells with optimal SPs is as follows.

Table 4. The Pre-Screening Analysis Matrix

\begin{tabular}{|c|c|c|c|c|c|}
\hline & $\mathrm{b}_{1}$ & $\mathrm{~b}_{2}$ & $\mathrm{~b}_{3}$ & $\mathrm{~b}_{4}$ & Supply \\
\hline$a_{1}$ & 0 & 0 & & & 20 \\
\hline$a_{2}$ & 0 & 0 & 0 & & 70 \\
\hline $\mathrm{a}_{3}$ & & & 0 & 0 & 30 \\
\hline $\mathrm{a}_{4}$ & & 0 & & 0 & 40 \\
\hline Demand & 50 & 30 & 30 & 50 & \\
\hline
\end{tabular}

Step 6 - 7: A study of Table 4 reveals that $a_{1}<b_{1}$. Therefore assign $X_{21}=\left(b_{1}-a_{1}\right)=30$. This is the minimum load value for this cell in any optimal solution. Similarly, $a_{3}<b_{4}$ and $a_{4}<b_{4}$. Assign loads $X_{44}=\left(b_{4}-a_{3}\right)=20$ and $X_{34}=$ $\left(b_{4}-a_{4}\right)=10$. Table 5 presents the modified pre-screening matrix after adjusting the demands and supplies where $\mathrm{x}$ marks the cells currently assigned with the required minimum load amounts.

Table 5. The Modified Pre-Screening Analysis Matrix

\begin{tabular}{|c|c|c|c|c|c|}
\hline & $\mathrm{b}_{1}$ & $\mathrm{~b}_{2}$ & $\mathrm{~b}_{3}$ & $\mathrm{~b}_{4}$ & Supply \\
\hline$a_{1}$ & 0 & 0 & & & 20 \\
\hline$a_{2}$ & $\mathrm{x}$ & 0 & 0 & & 40 \\
\hline$a_{3}$ & & & 0 & $\mathrm{x}$ & 20 \\
\hline $\mathrm{a}_{4}$ & & 0 & & $\mathrm{x}$ & 20 \\
\hline Demand & 20 & 30 & 30 & 20 & \\
\hline
\end{tabular}

Looking at Table 5, we see that $a_{3}<b_{3}$. Therefore assign $X_{23}=\left(b_{3}-a_{3}\right)=10$ as the minimum load. No further prescreening is possible for this load matrix. Table 6 presents the load assignment fixed due to the absolute and constraint structures of the TP to assign 90 units at the cost of $\$ 130$ and Table 7 presents the resultant floating loads matrix for the remaining 90 units.

Table 6. Fixed Load Assignment for an Optimal Solution

\begin{tabular}{|c|c|c|c|c|c|c|}
\hline & $\mathrm{b}_{1}$ & $\mathrm{~b}_{2}$ & $b_{3}$ & $\mathrm{~b}_{4}$ & $\mathrm{~b}_{5}$ & \multirow{2}{*}{$\begin{array}{c}\text { Supply } \\
40\end{array}$} \\
\hline \multicolumn{7}{|r|}{ (2) } \\
\hline $\mathrm{a}_{2}$ & 30 & & 10 & & & \\
\hline$a_{3}$ & & & & 10 & & 10 \\
\hline$a_{4}$ & & & & 20 & 20 & 40 \\
\hline Demand & 30 & & 10 & 30 & 20 & \\
\hline & & & 46 & & & \\
\hline
\end{tabular}


Table 7. Floating Loads Matrix for the Numerical Example

\begin{tabular}{|c|c|c|c|c|c|c|}
\hline \multirow{3}{*}{$\begin{array}{l}\mathrm{a}_{1} \\
\mathrm{a}_{2}\end{array}$} & $b_{1}$ & $b_{2}$ & $\mathrm{~b}_{3}$ & $\mathrm{~b}_{4}$ & $b_{5}$ & \multirow{2}{*}{$\begin{array}{c}\text { Supply } \\
20\end{array}$} \\
\hline & 0 & 0 & & & & \\
\hline & 0 & 0 & 0 & & & 30 \\
\hline $\mathrm{a}_{3}$ & & & 0 & 0 & & 20 \\
\hline$a_{4}$ & & 0 & & 0 & $\mathrm{X}$ & 20 \\
\hline Demand & 20 & 30 & 20 & 20 & $\mathrm{x}$ & \\
\hline
\end{tabular}

Step 8. A practitioner can now load the cells marked with zeros in any desired manner within the constraints of supply and demand. All solutions obtained in this manner are optimal. Two possible floating load assignments are as follows.

\begin{tabular}{|c|c|c|c|c|c|c|}
\hline \multirow[b]{2}{*}{$a_{1}$} & $\mathrm{~b}_{1}$ & $\mathrm{~b}_{2}$ & $b_{3}$ & $\mathrm{~b}_{4}$ & $b_{5}$ & \multirow{2}{*}{$\begin{array}{c}\text { Supply } \\
20\end{array}$} \\
\hline & & 20 & & & & \\
\hline$a_{2}$ & 20 & 10 & & & & 30 \\
\hline$a_{3}$ & & & 20 & & & 20 \\
\hline$a_{4}$ & & & & 20 & $\mathrm{x}$ & 20 \\
\hline \multirow[t]{2}{*}{ Demand } & 20 & 30 & 20 & 20 & $\mathrm{x}$ & \\
\hline & $\mathrm{b}_{1}$ & $\mathrm{~b}_{2}$ & $\mathrm{~b}_{3}$ & $\mathrm{~b}_{4}$ & $\mathrm{~b}_{5}$ & \multirow{6}{*}{$\begin{array}{c}\text { Supply } \\
20 \\
30 \\
20 \\
20\end{array}$} \\
\hline$a_{1}$ & & 20 & & & & \\
\hline$a_{2}$ & 20 & & 10 & & & \\
\hline$a_{3}$ & & & 10 & 10 & & \\
\hline$a_{4}$ & & 10 & & 10 & $\mathrm{x}$ & \\
\hline Demand & 20 & 30 & 20 & 20 & $\mathrm{x}$ & \\
\hline
\end{tabular}

Remark 1: The floating load distribution can be split into floating sub-distributions along different stepping stone chains. In our numerical example we can 'extract' the SS chain comprising four cells -- $(1,1),(1,2),(2,1)$, and $(2$, 2) -- and involving 50 units of load. After adjusting the supplies and demands the remaining cells will constitute the second floating sub-distribution involving a complex SS chain.

Finally, the two corresponding alternate optimal solutions are as follows:

Table 8. Alternate Optimal Solution 1 for the Numerical Example

\begin{tabular}{|c|c|c|c|c|c|c|}
\hline \multirow{3}{*}{$a_{1}$} & $\mathrm{~b}_{1}$ & $\mathrm{~b}_{2}$ & $\mathrm{~b}_{3}$ & $\mathrm{~b}_{4}$ & $\mathrm{~b}_{5}$ & \multirow{2}{*}{$\begin{array}{c}\text { Supply } \\
20\end{array}$} \\
\hline & & 20 & & & & \\
\hline & 50 & 10 & 10 & & & 70 \\
\hline$a_{3}$ & & & 20 & 10 & & 30 \\
\hline $\mathrm{a}_{4}$ & & & & 40 & 20 & 60 \\
\hline Demand & 50 & 30 & 30 & 50 & 20 & \\
\hline
\end{tabular}

Table 9. Alternate Optimal Solution 2 for the Numerical Example

\begin{tabular}{|c|c|c|c|c|c|c|}
\hline & $\mathrm{b}_{1}$ & $\mathrm{~b}_{2}$ & $\mathrm{~b}_{3}$ & $\mathrm{~b}_{4}$ & $\mathrm{~b}_{5}$ & Supply \\
\hline $\mathrm{a}_{1}$ & & 20 & & & & 20 \\
\hline $\mathrm{a}_{2}$ & 50 & & 20 & & & 70 \\
\hline $\mathrm{a}_{3}$ & & & 10 & 20 & & 30 \\
\hline $\mathrm{a}_{4}$ & & 10 & & 30 & 20 & 60 \\
\hline Demand & 50 & 30 & 30 & 50 & 20 & \\
\hline
\end{tabular}

Remark 2: In linear programming problems, if more than one optimal solution exists, then an infinite number of AOSs exist as any positive-weighted average of any two basic optimal solutions yields another AOS. The same 
does not always hold true for a TP due to integer restrictions on the load assignments. However, it may be possible to find some feasible AOSs by taking the positive-weighted average of the two optimal solutions of a TP. Since all load assignments in Table 8 and Table 9 are in the tens, any weighted average with a weight $\alpha / 10$, where $\alpha$ is an integer and $0 \leq \alpha \leq 10$ also provides an alternate optimal solution.

\section{CONCLUSION}

This paper expands on the theory of transportation problems beyond the material currently available in the textbooks and other sources. We introduce some of the already-known and some not-yet-known properties in a novel way, making them understandable to a wider spectrum of readers. The 'post optimal' analysis developed in the paper also provides a new view into the TP by demonstrating that some particular locations identified by the computational software as optimal can be loaded with greater or lesser amount or can even be omitted completely in an alternate equivalent solution.

We have also developed an algorithm that presents systematic steps to determine cells with fixed load (fixed due to the absolute structure of the TP) and some cells with minimal load (fixed due to the constraint structure of the TP). As a result, all floating cells are identified where a manager has the flexibility of allocation by identifying a minimum load for selected cells from the optimal solution, thereby empowering the manager to make load allocation decisions strategically, on the basis of an analysis of alternate assignments. The proposed algorithm can even allow omitting some of the routes identified originally as part of the optimal solution. The 'post solution' analysis can be a valuable tool for managers to assess 'parallel' solutions on the basis of an analysis of alternate load assignments.

\section{AUTHOR INFORMATION}

Veena Adlakha is a professor of Production Management at the University of Baltimore since 1984. She received her M.S. degree from Stanford University and the Ph.D. in Operations Research from the University of North Carolina at Chapel Hill. She has published her research in several prestigious journals including Networks, Management Science, Operations Research, and OMEGA. Dr. Adlakha served on the Southeast Asia Fulbright Peer Review Committee for 2002-2004. She has served as consultant to Verizon and BG\&E. Her current research interests include fixed-charge transportation problem, total quality management and web-based education.

Krzysztof Kowalski completed his undergraduate studies at Politechnika Gdanska, Poland in 1979 and M.S. degree in Engineering at the University of Mississippi in 1984. Between years 1984 and 1985 he worked as a full-time Research Assistant in the University of Arkansas. He was involved in computer modeling of groundwater movement. His work was published in Transactions of the American Society of the Agricultural Engineers and in Agricultural Water Management journal. Since 1986 he has worked in Connecticut Department of Transportation as a Transportation Engineer. His interests are optimization, linear programming and the theory of linear and nonlinear problems.

\section{REFERENCES}

1. Adlakha, V. \& Kowalski, K. (1999). An Alternative Solution Algorithm for Certain Transportation Problems, IJMEST 30, 719-728.

2. Adlakha, V., Kowalski, K. \& Lev, B. (2006). Solving Transportation Problems with Mixed Constraints, JMSEM 1, 47-52.

3. Arsham, H. (1992). Postoptimality Analyses of the Transportation Problem, J Opl Res Soc 43, 121-139.

4. Goyal, S.K. (1984). Improving VAM for the Unbalanced Transportation Problem, J Opl Res Soc 35, 11131114.

5. Intrator, J. \& Szwarc, W. (1988). An Inductive Property of Transportation Problem, Asia-Pacific J Opl Res Soc 5, 79-83.

6. Ramakrishnan, C.S. (1988). An Improvement to Goyal's Modified VAM for the Unbalanced Transportation Problem, J Opl Res Soc 39, 609-610. 
7. Sultan, A. (1988). Heuristic for Finding an Initial B. F. S. in Transportation Problems, Opsearch 25, $197-$ 199.

8. Sultan, A. \& Goyal, S.K. (1988). Resolution of Degeneracy in Transportation Problems, J Opl Res Soc 39, 411-413.

9. Wilsdon, C.E. (1990). A Simple, Easily Programmed Method for Locating Rook's Tours in the Transportation Problem, J Opl Res Soc 41, 879-880.

\section{NOTES}




\section{NOTES}

